\title{
A Lyotropic Inverse Ribbon Phase in a Branched-chain Polyoxyethylene Surfactant: Pressure Effects
}

\author{
Gemma C. Shearman ${ }^{\mathrm{a}}$, Nicholas J. Brooks ${ }^{\mathrm{a}}$, Gordon J.T. Tiddy ${ }^{\mathrm{b}}$, Michael Sztucki ${ }^{\mathrm{c}}$, Richard H. Templer ${ }^{\mathrm{a}}$, \\ Robert V. Law ${ }^{\mathrm{a}}$, Oscar Ces ${ }^{\mathrm{a}}$, John M. Seddon*a \\ 5 Received (in $X X X, X X X) X$ th $X X X X X X X X X 20 X X$, Accepted $X$ th $X X X X X X X X X 20 X X$ \\ DOI: 10.1039/b000000x
}

A centred-rectangular lyotropic inverse ribbon phase, rarely observed in inverse (type II) systems, has been found in the branched-chain polyoxyethylene surfactant tetradecyloctadecyl-tetraoxyethylene ether $\left(\mathrm{C}_{14} \mathrm{C}_{16} \mathrm{EO}_{4}\right)$ in excess water. This phase is stabilised by the application of hydrostatic pressure. The ratio 10 of the 2 -D cell parameters, $b / a$, is observed to be less than $\sqrt{3}$ (1.732) over the range of temperatures and pressures studied. The constructed pressure - temperature phase diagram shows that, at high temperatures or low pressures, the inverse ribbon phase converts into an inverse micellar cubic phase of spacegroup Fd3m, and at the opposite extreme, a lamellar gel phase was formed. The lattice parameters of the inverse ribbon phase were found to vary with pressure, with the structure becoming increasingly distorted away

15 from 2-D hexagonal symmetry $(b / a=\sqrt{ } 3)$ with increasing pressure.

\section{Introduction}

Amphiphiles are driven by the hydrophobic effect to selfassemble into a diverse range of aggregated phases when mixed with water. Three such assemblies are the fluid lamellar $\left(\mathrm{L}_{\alpha}\right), 2-\mathrm{D}$ 20 hexagonal $\left(\mathrm{H}_{\alpha}\right)$ and micellar / inverse micellar phases $\left(\mathrm{L}_{\mathrm{I}} / \mathrm{L}_{\mathrm{II}}\right)$, where the interfacial curvature in each case is uniform (and equal to zero for the $\mathrm{L}_{\alpha}$ phase). A number of lyotropic phases have been reported to exist between the lamellar and hexagonal phases, ranging from the 3-D bicontinuous cubics to the so-called

25 'intermediate' phases. ${ }^{1-3}$ Ribbon and mesh phases belong to the latter group, and can be considered as distorted hexagonal and perforated lamellar phases respectively, with non-uniform interfacial curvature. By tuning variables such as the temperature, pressure and amphiphile concentration, transitions between these 30 lyotropic liquid crystalline phases can be induced.

Ribbon phases are formed from flattened cylindrical or elliptical amphiphile aggregates, organized into 2-D periodic packed arrangements having oblique, primitive rectangular, centered rectangular (as shown in Fig. 1) or 2-D hexagonal symmetry. ${ }^{4,5}$

35 Although observed in several 'normal' (type I) surfactant-water systems, where the interface is curved away from the aqueous medium (e.g. some polyoxyethylene cholesteryl ether/water systems $^{6,7}$ ), the ribbon phase has rarely been seen in 'inverse' (type II) systems, where the interfacial curvature is towards the 40 water regions; those systems that do form inverse ribbon $\left(\mathrm{Rb}_{\mathrm{II}}\right)$ phases only tend to do so in extremely dehydrated conditions. ${ }^{8-12}$ However, since the inverse ribbon and inverse hexagonal $\left(\mathrm{H}_{\mathrm{II}}\right)$ phases have been associated with cellular processes that involve a disruption of the cell membrane improving the current 45 understanding of these structures and any phase transitions that they might undergo is of great potential importance. Recently, Kent et al. investigated the structural dependence on temperature

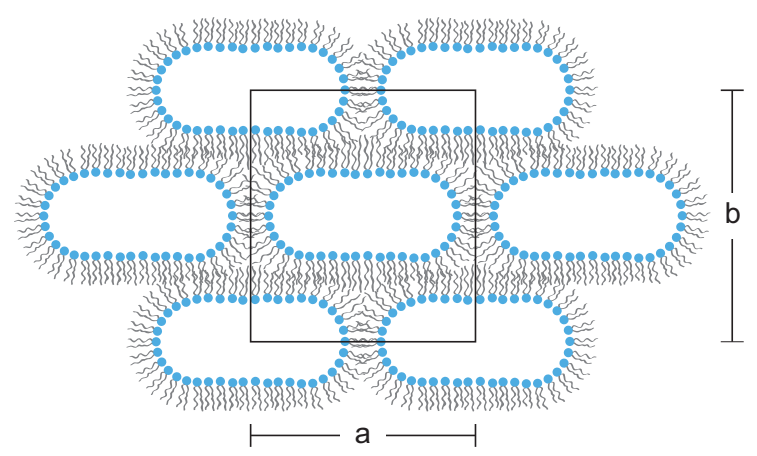

Fig. 1 A cross section of a centred-rectangular inverse lyotropic ribbon phase of $\mathrm{cmm}$ symmetry, where $a$ and $b$ are the cell parameters.

of the inverse ribbon phase formed by a low hydration mixture of dioleoylphosphatidylcholine and dioleoylphosphatidylethanolamine, and they reported that the distortion away from the 2-D hexagonal symmetry characteristic 55 of the inverse hexagonal phase became more pronounced with decreasing temperature. ${ }^{12}$

By adjusting both the temperature and pressure of a branchedchain polyoxyethylene surfactant in water, we show that it is possible to form the inverse ribbon phase in excess aqueous ${ }_{60}$ conditions, and we present data showing the variation in the lattice parameters with pressure.

\section{Materials and Methods}

\section{Sample Preparation}

The branched-chain polyoxyethylene surfactant ${ }_{65} \mathrm{C}_{16} \mathrm{H}_{33} \mathrm{CH}\left(\mathrm{C}_{14} \mathrm{H}_{29}\right) \mathrm{CH}_{2}\left(\mathrm{OC}_{2} \mathrm{H}_{4}\right)_{4} \mathrm{OH}$ (tetradecyloctadecyltetraoxyethylene-ether; $\mathrm{C}_{14} \mathrm{C}_{16} \mathrm{EO}_{4}$ ), with a purity of $97 \%$, was a 
<smiles>[R]C(C)COCCOCCO</smiles>

$\mathrm{R}_{2}$

Scheme 1 The generic structure of the branched-chain polyoxyethylene surfactant studied here, where $\mathrm{R}_{1}$ and $\mathrm{R}_{2}$ are saturated alkyl chains.

gift from Unilever plc, and was used as received. The surfactant 5 was lyophilized and then mixed with HPLC grade water (VWR; Poole, U.K.) to obtain samples with a final water content of $66 \mathrm{wt}$ $\%$. This ensured that the samples were in excess water over the range of temperatures and pressures studied. The samples were then homogenized by mechanically mixing with a spatula and 10 repeated temperature cycling; specifically, each sample was heated from $-20{ }^{\circ} \mathrm{C}$ to above $50{ }^{\circ} \mathrm{C}$ and then cooled back down to $-20{ }^{\circ} \mathrm{C}$ ten times to drive the sample through all of the phase boundaries of interest. The sample was then stored at $-20{ }^{\circ} \mathrm{C}$ until examination, when it was placed in a Teflon ring of $1 \mathrm{~mm}$ 15 thickness, sealed by two Mylar windows that were attached to the Teflon disk by double-sided sticky tape.

\section{Synchrotron Small-Angle X-Ray Scattering (SAXS)} Measurements.

Experiments were carried out at the ID02 high brilliance 20 beamline at the European Synchrotron Radiation Facility (ESRF) in Grenoble (France), using a custom-built high pressure apparatus capable of applying hydrostatic pressures of between 1 and 4000 bar to the sample, with an accuracy of \pm 10 bar. This apparatus was designed, built and generously provided by 25 Professor R. Winter, University of Dortmund, and is described in detail by Woenckhaus et al. ${ }^{13}$ The temperature of the high pressure cell was controlled with a precision of $\pm 0.2^{\circ} \mathrm{C}$ by means of a thermostatable jacket through which circulating water was pumped continuously. The synchrotron beamline was tuned 30 to deliver a photon energy of $16.5 \mathrm{keV}$ (corresponding to a wavelength of $0.7515 \AA$ ), with beam dimensions of approximately $200 \times 400 \mu \mathrm{m}$ and a typical flux of $3 \times 10^{13}$ photons/s. Diffraction patterns were recorded using a fiberoptically coupled FReLoN (Fast-Readout, Low Noise) CCD

35 detector, with a sample-detector distance of $2.5 \mathrm{~m}$. The 2-D images were converted to 1-D patterns and subsequently analysed using AXcess, a software package based on IDL written by Dr Andrew Heron. ${ }^{14}$

\section{Results and Discussion}

\section{${ }_{40}$ Ribbon phase identification}

Polyoxyethylene surfactants have been found to exhibit a wide range of lyotropic liquid crystalline phase behavior, and have been widely studied as they are extensively used in the chemical industry. Branched-chain polyoxyethylene surfactants (Scheme 1) 45 are predicted to exhibit inverse lyotropic phases, due to their bulky hydrophobic region. Fig. 2 shows a sequence of integrated 1-D SAXS patterns of $\mathrm{C}_{14} \mathrm{C}_{16} \mathrm{EO}_{4}$ in excess water at $17{ }^{\circ} \mathrm{C}$ upon increasing the applied hydrostatic pressure systematically from atmospheric pressure to 3000 bar. Three different phases can ${ }_{50}$ clearly be seen, with the first phase transition occurring at approximately 300 bar and the second, with a large region of

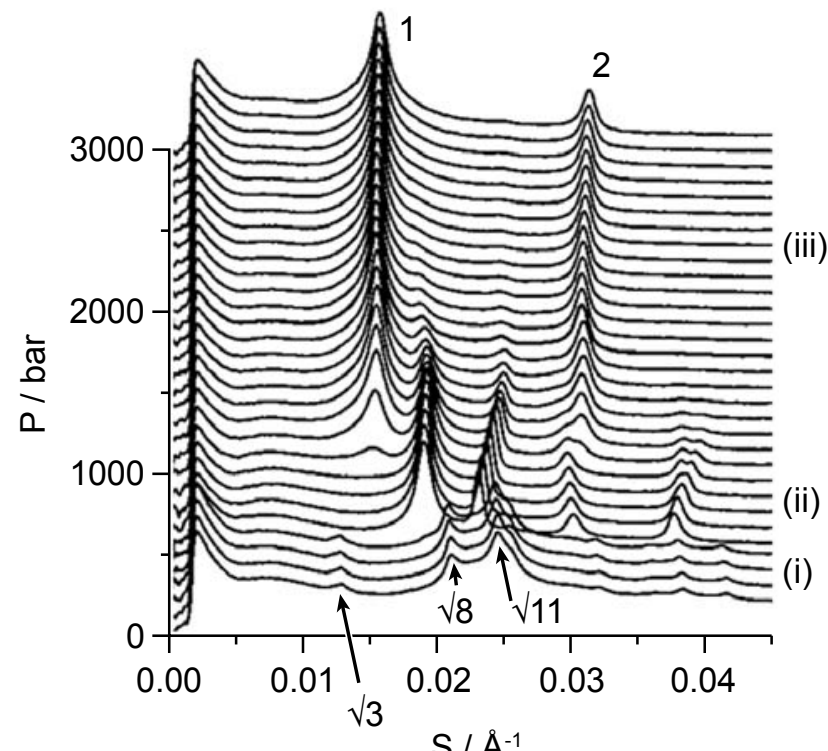

$S / \AA^{-1}$

Fig. 2 Small-angle $X$-ray diffraction patterns from $\mathrm{C}_{14} \mathrm{C}_{16} \mathrm{EO}_{4}$ in excess water recorded over a range of pressures (from $1-3000 \mathrm{bar}$ ), where the 55 intensity profiles are shown on a logarithmic scale. A number of the Bragg peaks for the inverse micellar cubic $\mathrm{Fd} 3 \mathrm{~m}$ phase in region (i), and the lamellar phase in region (iii), have been labeled for clarity. A 2-D centred rectangular phase is observed in region (ii). Note, the $\mathrm{z}$ axis is not shown for clarity; it represents $\log$ (intensity) in arbitrary units.

60 coexistence, between $\sim 1000-2000$ bar. The mesophase existing at low pressures (region (i) of Fig. 2) is an inverse micellar cubic phase of Fd3m symmetry (with Bragg peaks corresponding to the reflections $111,220,311,222,331,422,511 / 333$ and 440 being observed). The reflections in region (iii) of Fig. 2 correspond to a ${ }_{65}$ lamellar $\mathrm{L}_{\beta}$ gel phase (see Supporting Information (SI) for a full description of the wide-angle X-ray scattering (WAXS) characterisation. Identification as a gel phase is based on a single, symmetrical peak close to $4.2 \AA$ ).

At low pressures, four reflections in region (ii) are observed; it is 70 common to observe only a few diffraction peaks from liquid crystalline phases due to liquid-like short range positional order. As the pressure is increased the fourth Bragg peak splits into two separate peaks. Although five diffraction peaks are still relatively few, they can be consistently indexed as a 2-D rectangular phase, 75 where for a 2-D rectangular lattice the reflections are determined by:

$$
\frac{1}{d_{h k l}^{2}}=\frac{h^{2}}{a^{2}}+\frac{k^{2}}{b^{2}}
$$

where $h$ and $k$ are Miller indices, and $a$ and $b$ are the lattice parameters of the rectangular unit cell (see Fig. 1) for which we 80 have defined $b>a$.

Plane groups pmm, pgg and $\mathrm{cmm}$ would all be consistent with such reflections. However, by focusing on their reflection conditions, it has been shown that it is possible to identify the appropriate lattice symmetry with only a few reflections due to 85 the high experimental resolution. ${ }^{15,16}$ The restrictions for the $p g g$ plane group are: for $h 0: h=2 n$ and for $0 k: k=2 n$, whereas for the primitive $p m m$ plane group there are no restrictions on the reflections. Therefore, in the former case we would expect to see 
Table 1 Experimental and calculated Bragg spacings of the centered rectangular ribbon phase, and derived lattice parameters for $\mathrm{C} 14 \mathrm{C} 16 \mathrm{EO} 4$ in excess water at $17^{\circ} \mathrm{C}$, as a function of pressure.

\begin{tabular}{|c|c|c|c|c|c|c|c|c|c|c|c|c|c|}
\hline \multirow[t]{2}{*}{$\mathbf{P} /$ bar $^{a}$} & \multicolumn{2}{|c|}{$d_{11} / \AA$} & \multicolumn{2}{|c|}{$\mathbf{d}_{02} / \AA$} & \multicolumn{2}{|c|}{$\mathbf{d}_{20} / \AA$} & \multicolumn{2}{|c|}{$\mathbf{d}_{22} / \AA$} & \multicolumn{2}{|c|}{$\mathrm{d}_{13} / \AA$} & \multirow[t]{2}{*}{$\mathbf{a} / \AA^{b}$} & \multirow[t]{2}{*}{$\mathbf{b} / \AA^{b}$} & \multirow[t]{2}{*}{$\mathbf{b} / \mathbf{a}$} \\
\hline & (exp) & (calc) & (exp) & (calc) & (exp) & (calc) & (exp) & (calc) & (exp) & (calc) & & & \\
\hline 200 & 53.50 & 53.50 & 44.45 & 44.47 & & & & & & & 66.98 & 88.93 & 1.328 \\
\hline 300 & 53.64 & 53.68 & 44.14 & 44.15 & 33.78 & 33.80 & 26.96 & 26.84 & & & 67.60 & 88.30 & 1.306 \\
\hline 400 & 54.10 & 54.08 & 43.91 & 43.90 & 34.34 & 34.33 & 26.98 & 27.04 & & & 68.65 & 87.80 & 1.279 \\
\hline 500 & 54.18 & 54.13 & 43.48 & 43.46 & 34.62 & 34.59 & 26.90 & 27.06 & & & 69.18 & 86.92 & 1.256 \\
\hline 600 & 54.20 & 54.20 & 43.13 & 43.16 & 34.81 & 34.82 & 27.09 & 27.10 & 26.65 & 26.59 & 69.63 & 86.32 & 1.240 \\
\hline 700 & 54.17 & 54.16 & 42.70 & 42.71 & 35.00 & 35.02 & 27.07 & 27.08 & 26.39 & 26.38 & 70.03 & 85.42 & 1.220 \\
\hline 800 & 53.96 & 53.98 & 42.22 & 42.23 & 35.09 & 35.09 & 27.01 & 26.99 & 26.16 & 26.13 & 70.18 & 84.46 & 1.203 \\
\hline 900 & 53.84 & 53.80 & 41.76 & 41.82 & 35.09 & 35.13 & 26.88 & 26.90 & 25.98 & 25.91 & 70.26 & 83.64 & 1.190 \\
\hline 1000 & 53.77 & 53.77 & 41.51 & 41.55 & 35.25 & 35.27 & 26.92 & 26.89 & 25.84 & 25.78 & 70.54 & 83.09 & 1.178 \\
\hline 1100 & 53.77 & 53.73 & 41.30 & 41.36 & 35.28 & 35.34 & 26.93 & 26.86 & 25.73 & 25.68 & 70.67 & 82.71 & 1.170 \\
\hline 1200 & 53.79 & 53.78 & 41.07 & 41.07 & & & & & & & 71.16 & 82.14 & 1.154 \\
\hline 1300 & 53.78 & 53.78 & 40.85 & 40.86 & & & & & & & 71.44 & 81.71 & 1.144 \\
\hline 1400 & 53.84 & 53.84 & 40.64 & 40.64 & & & & & & & 71.88 & 81.27 & 1.131 \\
\hline 1500 & 53.86 & 53.86 & 40.48 & 40.48 & & & & & & & 72.14 & 80.96 & 1.122 \\
\hline 1600 & 53.86 & 53.86 & 40.34 & 40.34 & & & & & & & 72.35 & 80.68 & 1.115 \\
\hline 1700 & 53.94 & 53.94 & 40.22 & 40.22 & & & & & & & 72.71 & 80.43 & 1.106 \\
\hline
\end{tabular}

${ }^{a}$ At 200 bar, the ribbon phase co-exists with an Fd3m cubic phase; between 800 and 2000 bar, the ribbon phase and gel phase co-exist.

${ }^{b}$ The cell parameters of the ribbon phase were determined using an iterative procedure (see ESI for details).

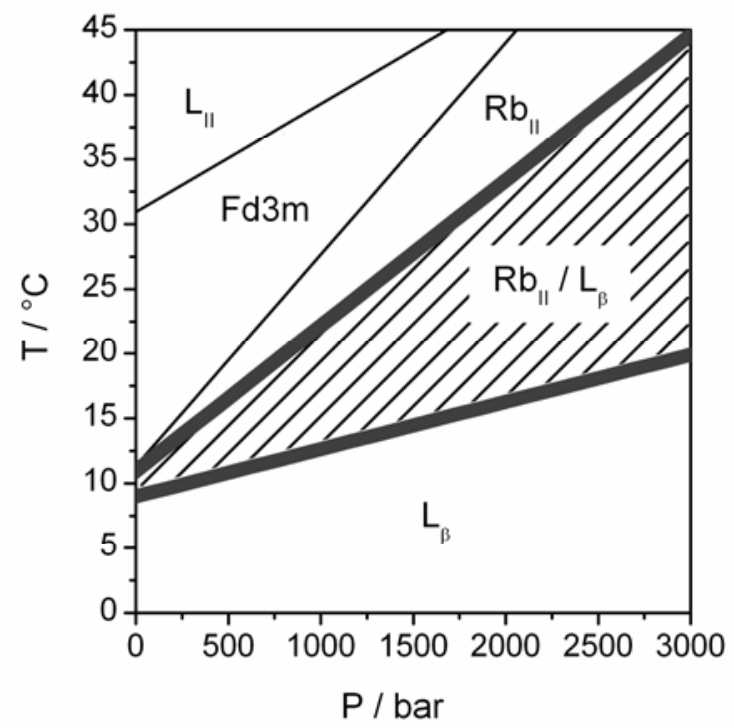

Fig. 3 The experimental temperature-pressure phase diagram for $\mathrm{C}_{14} \mathrm{C}_{16} \mathrm{EO}_{4} / 66 \mathrm{wt} \%$ water, where the region of $\mathrm{Rb}_{\mathrm{II}} / \mathrm{L}_{\beta}$ phase coexistence is hatched for clarity.

reflections at, for example, $45.90 \AA$ and $32.28 \AA$ corresponding to ${ }_{10} d_{12}$ and $d_{21}$ at a pressure of $600 \mathrm{bar}$; for the latter plane group the reflections at $54.2 \AA$ and $43.13 \AA$ would then correspond to $\mathrm{d}_{01}$ and $d_{10}$ for which $d_{11}$ is predicted to lie at $33.75 \AA$. As these reflections are not seen, the $\mathrm{cmm}$ plane group, for which the restriction on the Bragg reflections is given by $h+k=2 n$, is 15 most consistent with the observed reflections. The observed and calculated $d_{h k}$ reflections observed for $\mathrm{C}_{14} \mathrm{C}_{16} \mathrm{EO}_{4}$ in excess water at $17{ }^{\circ} \mathrm{C}$ are listed in Table 1 , together with their assigned values assuming centered rectangular $(\mathrm{cmm})$ symmetry. From Table 1 it is clear that for each measurement the ratio of the cell parameters 20 (b/a) falls within the range $1<b / a<\sqrt{3}$, which can be envisaged as a deformation of a 2-D hexagonal mesophase that has been stretched in the $a$ direction and/or compressed in the $b$ direction. Although inverse lyotropic ribbon phases have been previously reported, to the best of our knowledge, $b / a$ has always been 25 reported as being greater than $\sqrt{3}$, i.e. the deformation of the hexagonal lattice has occurred in the opposite manner from that observed here. However, a similar type of deformation has been reported for an inorganic mesophase formed by the calcification of a silica precursor around a templating agent. ${ }^{17-19}$

\section{${ }_{30}$ Structural and lyotropic phase behavior}

The experimental temperature-pressure phase diagram of $\mathrm{C}_{14} \mathrm{C}_{16} \mathrm{EO}_{4}$ in excess (66 wt $\%$ ) water is shown in Fig. 3, over temperature and pressure ranges of $0-45^{\circ} \mathrm{C}$ and $1-3000$ bar, respectively.

${ }_{35}$ In Fig. 3, the phases are shown to appear in the order $\mathrm{L}_{\beta} \rightarrow$ coexisting $\mathrm{Rb}_{\mathrm{II}} / \mathrm{L}_{\beta} \rightarrow \mathrm{Rb}_{\mathrm{II}} \rightarrow \mathrm{Fd} 3 \mathrm{~m} \rightarrow \mathrm{L}_{\mathrm{II}}$ with either increasing temperature or decreasing pressure. This means that for this system, by increasing the pressure one can stabilize, for example, the $\mathrm{Rb}_{\text {II }}$ phase with respect to the $F d 3 m$ phase. This phase 40 sequence is typical of lyotropic liquid crystalline phase behaviour, in that more highly curved phases are normally obtained by increasing the temperature, or by decreasing the pressure. However, the Fd3m phase can also be stabilised with respect to the inverse micellar $\mathrm{L}_{\mathrm{II}}$ phase by decreasing ${ }_{45}$ temperature or increasing pressure, and this is presumably not 


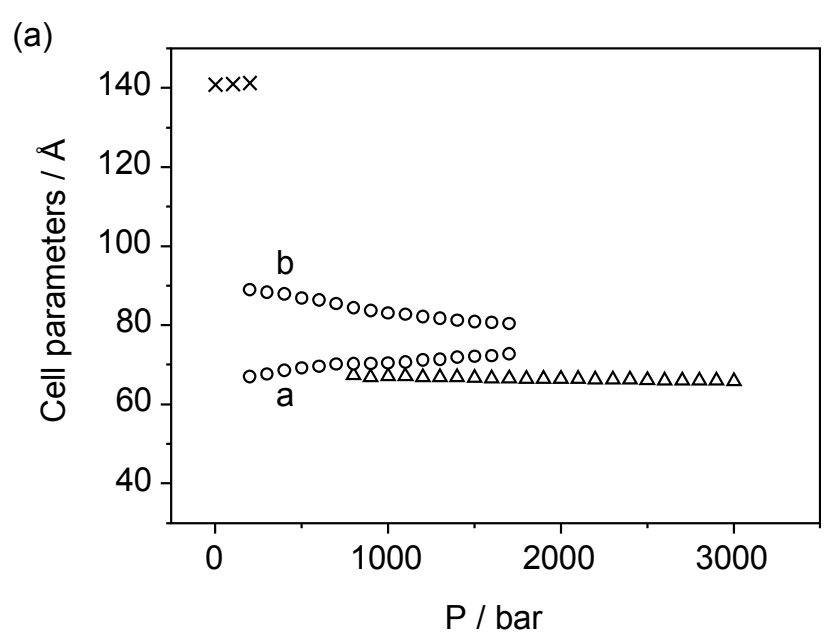

(b)

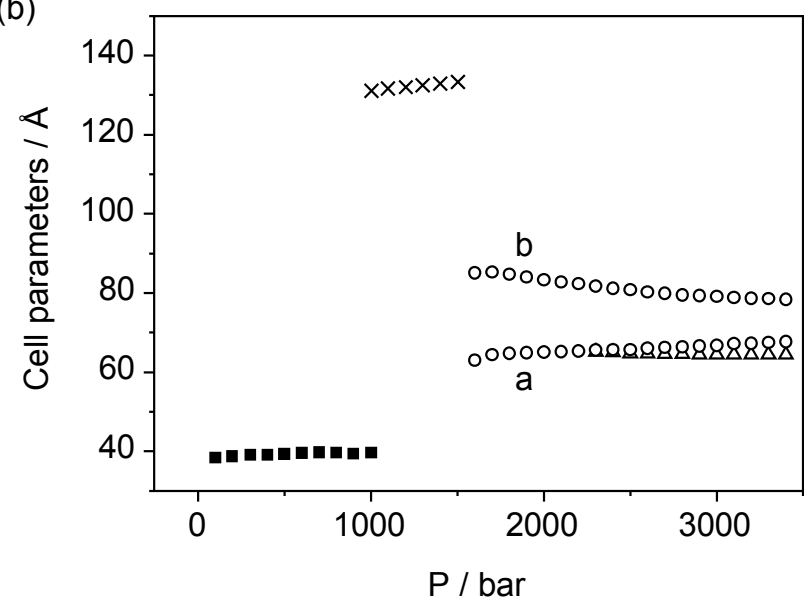

Fig. 4 The effect of pressure on the lattice parameters of $\mathrm{C}_{14} \mathrm{C}_{16} \mathrm{EO}_{4}$ in excess water, at (a) $17^{\circ} \mathrm{C}$, and (b) $37^{\circ} \mathrm{C}$, with the $\mathrm{L}_{\mathrm{II}}, F d 3 m, \mathrm{Rb}_{\mathrm{II}}$ and $\mathrm{L}_{\beta}$ phases denoted by solid squares, crosses, open circles and open triangles,

5 respectively. Two sets of open circles are shown at each pressure for which the $\mathrm{Rb}_{\text {II }}$ phase was observed, denoting the $a$ and $b$ cell parameters. The spacings plotted for the inverse micellar solution $\mathrm{L}_{\mathrm{II}}$ correspond to the position of the maximum of the small-angle diffuse peak from this phase.

10 due to a difference in interfacial curvature, but rather reflects a greater disorder in the latter phase. At atmospheric pressure the ribbon phase exists over a temperature range of only $1{ }^{\circ} \mathrm{C}$ or less, but the range of stability increases markedly with increasing pressure. This is due to the pressure-dependence of the transition 15 temperatures for different phases being markedly dissimilar: a slope of about $16{ }^{\circ} \mathrm{C} / \mathrm{kbar}$ was observed for the $\mathrm{Fd} 3 \mathrm{~m}-\mathrm{Rb}_{\text {II }}$ phase boundary, which is within the typical range of phase boundary gradients for saturated-chain amphiphiles, ${ }^{20,21}$ whereas for the $\mathrm{Rb}_{\mathrm{II}}$ - coexisting $\mathrm{Rb}_{\mathrm{II}} / \mathrm{L}_{\beta}$ and in particular the $\mathrm{Rb}_{\mathrm{II}} / \mathrm{L}_{\beta}$ ${ }_{20} \mathrm{~L}_{\beta}$ boundaries, the gradients are much smaller. Due to the inherent difficulty in determining an exact gradient of a phase boundary associated with a region of phase co-existence, in Fig. 3 these two phase boundaries are represented by thick dark grey lines to indicate their tentative nature.

${ }_{25}$ As noted earlier, ribbon phases have been reported previously in mixed surfactant system where the surfactant composition varies between the curved and flat areas of the ribbon. Here we have demonstrated ribbon phase formation using only one surfactant and it is likely that adoption of a ribbon structure in this case
30 allows the systerm to achieve an average interfacial mean curvature close to the surfactants spontaniour curvature. While the same average mean curvature could in principle be achieved in a very highly hydrated $\mathrm{H}_{\text {II }}$ phase, this would lead to large voids forming in the hydrophobic region which could not be filled by

35 the surfactant's hydrocarbon chains. The lattice parameter values given in Table 1 show that increasing pressure leads to a decrease in the average interfacial curvature reflecting the expected pressure induced change in the surfactant's spontanious curvature. The molecular achitecture of the surfactant used here 40 may be key to allowing it to adopt different curvatures as the relatively flexible head group can sample different conformational states.

It should be noted that the presence of a $\mathrm{Rb}_{\mathrm{II}} / \mathrm{L}_{\beta}$ phase coexistence region is not strictly speaking allowed by the Gibbs phase rule

45 (this can be considered to be a single component system as the water is in excess). However, we have observed this coexistence region consistently in a number of independent samples. We believe that this does not represent the true thermodynamic equilibrium state as time-resolved experiments show the peaks 50 assigned to the $\mathrm{L}_{\beta}$ phase decaying very slowly with time within the coexistence region. Hence we have presented this as an experimental phase diagram.

The pressure dependence of the lattice parameters of the phases formed by $\mathrm{C}_{14} \mathrm{C}_{16} \mathrm{EO}_{4}$ in excess water at two different 55 temperatures are shown in Fig. 4 . At $37{ }^{\circ} \mathrm{C}$, the lattice parameter of the $\mathrm{Fd} 3 \mathrm{~m}$ phase was found to increase with pressure with a gradient of $4.2 \AA \mathrm{kbar}^{-1}$. The approximately linear trend displayed by the Fd3m phase was typical of all the phases observed in this study. This observed increase in lattice constant with pressure is 60 typical for inverse curved phases. ${ }^{20,22}$ However, a small opposite effect was observed for the lamellar gel phase, where $(\mathrm{d} a / \mathrm{d} p)_{17^{\circ} \mathrm{C}}$ and $(\mathrm{d} a / \mathrm{d} p)_{37^{\circ} \mathrm{C}}$ were found to be $-0.53 \AA \mathrm{kbar}^{-1}$ and $-0.73 \AA \mathrm{kbar}$ 1 . This could be due to a small tilting of the alkyl chains, driven by an increased headgroup hydration, with pressure. Interestingly, ${ }_{65}(\mathrm{~d} a / \mathrm{d} p)_{T}$ was not found to be significantly affected by the coexistence of the $\mathrm{Rb}_{\text {II }}$ phase although this is not the case for the $\mathrm{Rb}_{\text {II }}$ phase itself, with $(\mathrm{d} a / \mathrm{d} p)_{T}$ being smaller when the ribbon phase is in a biphasic region. Specifically, for e.g. $37{ }^{\circ} \mathrm{C}$, $\left(\mathrm{d} a\left(R b_{I I}\right) / \mathrm{d} p\right)=+4.02 \AA \mathrm{kbar}^{-1}$ and $\left(\mathrm{d} b\left(R b_{I I}\right) / \mathrm{d} p\right)=-4.5 \AA \mathrm{kbar}^{-1}$ 70 but when in co-existence with the lamellar gel phase, $\left(\mathrm{d} a\left(R b_{I I}\right) / \mathrm{d} p\right)=+2.09 \AA \mathrm{kbar}^{-1}$ and $\left(\mathrm{d} b\left(R b_{I I}\right) / \mathrm{d} p\right)=-3.7 \AA \mathrm{kbar}^{-1}$; these gradients increase at lower temperatures, which is also reflected in the dependence of the ratio of the cell parameters, with $(\mathrm{d}(\mathrm{b} / \mathrm{a}) / \mathrm{d} p)_{17^{\circ} \mathrm{C}}=-0.23 \mathrm{kbar}^{-1}\left(-0.07 \mathrm{kbar}^{-1}\right.$ in the biphasic 75 region) and with $(\mathrm{d}(b / a) / \mathrm{d} p)_{37^{\circ} \mathrm{C}}=-0.15 \mathrm{kbar}^{-1}\left(-0.08 \mathrm{kbar}^{-1}\right.$ in the biphasic region). The decrease in $b / a$ reveals that the system is being driven further away from the ideal 2-D hexagonal packing (for which $b / a=\sqrt{3}$ ) with increasing pressure. A similar observation has recently been reported for a low hydration 80 dioleoylphosphatidylcholine: dioleoylphosphatidylethanolamine mixture, but for decreasing temperature (known to act in an opposite manner to pressure). ${ }^{12}$

\section{Conclusions}

We have shown that, for a branched-chain non-ionic surfactant, 85 an inverse ribbon phase can exist in highly hydrated conditions and that the region of its phase stability can be increased by the 
application of pressure, at the expense of the inverse micellar cubic phase. The destabilisation of the $F d 3 m$ phase with pressure is predictable, as previous work has provided evidence that phases with reduced chain splay are adopted at high pressures ${ }^{20}$. ${ }_{5}$ However, one might expect a transition from the $\mathrm{Fd} 3 \mathrm{~m}$ phase to the inverse hexagonal $\mathrm{H}_{\text {II }}$ phase, rather than the inverse ribbon $\mathrm{Rb}_{\text {II }}$ phase, to be observed. Indeed, recently ${ }^{23}$ we have shown that application of pressure to a hydrated binary lipid mixture of dioleoyl-phosphatidylcholine (DOPC) and dioleoyl-glycerol

10 (DOG) in the Fd3m cubic phase, induces a sharp transition at a critical pressure (close to 2000 bar) to a phase separated mixture of an $\mathrm{H}_{\text {II }}$ phase and ordered lamellar phase; such a pressure induced phase separation is not possible in the single surfactant system studied in the present work. Here, the preferred stability 15 of the inverse ribbon phase, with non-uniform interfacial curvature, suggests that the polyoxyethylene surfactant may preferentially adopt different conformations in the differentlycurved regions of the inverse ribbons; further research is needed to confirm this hypothesis.

\section{${ }_{20}$ Acknowledgements}

We thank Unilever plc. for the gift of the surfactant, and the UK Engineering and Physical Sciences Research Council (EPSRC) for the award of Platform Grant EP/G00465X. We acknowledge the European Synchrotron Radiation Facility for provision of 25 their facilities. We are grateful to Dr. Theyencheri Narayanan and Mr. Christophe Jeworrek for their assistance with the experiments at beamline ID02. We thank Professor Roland Winter (University of Dortmund) for the use of the pressure-jump apparatus.

\section{Notes}

$30{ }^{a}$ Department of Chemistry, Imperial College London, SW7 2AZ, U.K. * E-mail: j.seddon@imperial.ac.uk

${ }^{b}$ School of Chemical Engineering \& Analytical Science, University of Manchester, PO Box 88, Manchester, M60 1QD, U.K.

${ }^{c}$ ESRF, BP 220, F-38043, Grenoble, France

$35 \dagger$ Electronic Supplementary Information (ESI) available: Wide-angle Xray scattering methodology and identification of the lamellar gel phase; iterative procedure used to determine the cell parameters of the inverse ribbon phase. See DOI: 10.1039/b000000x/

\section{References}

40 1. M. C. Holmes, Curr. Opin. Colloid Interface Sci., 1998, 3, 485-492.

2. S. T. Hyde and G. E. Schröder, Curr. Opin. Colloid Interface Sci., 2003, 8, 5-14.

3. M. C. Holmes and M. S. Leaver, in Bicontinuous Liquid Crystals, Crc Press-Taylor \& Francis Group, Boca Raton, 2005, vol. 127, pp. 15-39.

4. H. Hagslatt, O. Soderman and B. Jonsson, Liq. Cryst., 1992, 12, 667688.

5. H. Hagslatt, O. Soderman and B. Jonsson, Liq. Cryst., 1994, 17, 157177.

50 6. T. Sato, M. K. Hossain, D. P. Acharya, O. Glatter, A. Chiba and H. Kunieda, J. Phys. Chem. B, 2004, 108, 12927-12939.

7. M. K. Hossain, D. P. Acharya, T. Sakai and H. Kunieda, J. Colloid Interface Sci., 2004, 277, 235-242.
8. A. E. Skoulios and V. Luzzati, Acta Crystallographica, 1961, 14, 278-286.

9. W. Pohle and C. Selle, Chem. Phys. Lipids, 1996, 82, 191-198.

10. E. Y. Shalaev and P. L. Steponkus, Biochim. Biophys. Acta, 1999, 1419, 229-247.

11. E. Y. Shalaev and P. L. Steponkus, Biochim. Biophys. Acta, 2001, 1514, 100-116

12. B. Kent, C. J. Garvey, D. Cookson and G. Bryant, Chem. Phys. Lipids, 2009, 157, 56-60.

13. J. Woenckhaus, R. Kohling, R. Winter, P. Thiyagarajan and S. Finet, Rev. Sci. Instrum., 2000, 71, 3895-3899.

65 14. J. M. Seddon, A. M. Squires, C. E. Conn, O. Ces, A. J. Heron, X. Mulet, G. C. Shearman and R. H. Templer, Philos. Trans. R. Soc. London, A, 2006, 364, 2635-2655.

15. Y. Hendrikx and J. Charvolin, Liq. Cryst., 1992, 11, 677-698.

16. S. Gustafsson and P. O. Quist, J. Colloid Interface Sci., 1996, 180, $70 \quad 564-573$

17. D. Y. Zhao, Q. S. Huo, J. L. Feng, J. M. Kim, Y. J. Han and G. D. Stucky, Chem. Mater., 1999, 11, 2668-2672.

18. A. F. Gross, V. H. Le, B. L. Kirsch and S. H. Tolbert, Langmuir, 2001, 17, 3496-3504.

75 19. J. El Haskouri, S. Cabrera, M. Caldes, C. Guillem, J. Latorre, A. Beltran, D. Beltran, M. D. Marcos and P. Amoros, Chem. Mater., 2002, 14, 2637-2643.

20. P. M. Duesing, J. M. Seddon, R. H. Templer and D. A. Mannock, Langmuir, 1997, 13, 2655-2664.

80 21. R. Winter, Biochim. Biophys. Acta, 2002, 1595, 160-184.

22. M. Pisani, T. Narayanan, G. M. Di Gregorio, C. Ferrero, S. Finet and P. Mariani, Phys. Rev. E, 2003, 68, 021924.

23. A. I. I. Tyler, G. C. Shearman, N. J. Brooks, H. Delacroix, R. V. Law, R. H. Templer, O. Ces and J. M. Seddon, Phys. Chem. Chem. Phys., 2011, DOI: 10.1039/c0cp01783c 